\title{
Improvement of Chemical and Thermal Properties of Polyethylene Terephthalate (PET) by Using Multi-walled Carbon Nanotubes (MWCNTs)
}

\author{
Eman Mohammed Khalaf, Sameer Ahmed Awad \\ Department of Chemistry, University of Al-Anbar, Ramadi, Iraq \\ Email address: \\ Emanmohammed467@yahoo.com (E. M. Khalaf), Sameer.msc1981@gmail.com (S. A. Awad) \\ To cite this article: \\ Eman Mohammed Khalaf, Sameer Ahmed Awad. Improvement of Chemical and Thermal Properties of Polyethylene Terephthalate (PET) by \\ Using Multi-walled Carbon Nanotubes (MWCNTs). International Journal of Materials Science and Applications. \\ Vol. 5, No. 6, 2016, pp. 297-301. doi: 10.11648/j.ijmsa.20160506.20
}

Received: October 15, 2016; Accepted: November 14, 2016; Published: December 17, 2016

\begin{abstract}
This work aims to investigate the improvements of thermal and chemical properties of polyethylene terephthalate (PET) by using Multi-walled carbon nanotubes (MWCNTs). MWCNT were used as a reinforcement in polyethylene terephthalate with different weight percentages $(0.1,0.5$, and 1$) \%$ respectively. The direct mixing process was used to prepare (polyethylene terephthalate / MWCNTs) composites. The ultrasonic mixing technique was applied to disperse the MWCNTs material into polyethylene terephthalate. The intensities of absorption of hydroxyl and carbonyl group decreased with increasing the addition of MWCNTs from (0.1-1\%), comparing with pure PET. The results of TGA showed that the incorporation of MWCNTs into pure PET slightly improves the thermal stability because interfaces between multifuntionlized MWCNTs and pure PET causes of reducing brittle behavior and increased due to the high crosslinking of pure PET. The mechanical tests appeared significant improvements with increasing of MWCNTs concentrations. The SEM micrographs showed more voids between the pure PET chains before adding MWCNTs while MWCNTs-PET composites appeared more homogeneous, crosslink density and less voids between the chains.
\end{abstract}

Keywords: Thermal Stability, Polyethylene Terephthalate, MWCNTs, TGA, FTIR, SEM

\section{Introduction}

Polymer nanocomposites are a new type of composite materials, and in the recent decade, many nanoparticles such as multi-walled carbon nanotubes (MWCNTs), and graphene have been applied in the synthesis of PET nanocomposites via melt mixing or polymerization. They are prepared by the incorporation of carbon nanofiber fillers to improve the mechanical and electrical properties with chemical stability by a very attractive way [1-6]. Poly (ethylene terephthalate) is a thermoplastic semicrystalline and one of the aromatic polyesters that widely utilized in the textile industry and beverage bottles, because of its first high elastic modulus, strength, toughness, low cost, high transparency, high processability, and moderate recyclability. Thus, it is widely utilized in several industrial applications such as liquid bottles, fibers, films and engineering plastics [7-17]. Carbon nanotubes
(CNTs) as nanofillers in polymeric materials are the most of the relevant materials of the recent century.

CNTs have confirmed the high potential and effective reinforcing fillers for industrial applications, and they can be on two types multi-walled (MWCNTs) and singlewalled (SCNTs) and are used as fillers for many types of plastic to improve the mechanical, thermal, optical, electrical and chemical properties [18-21]. Iijima discovered carbon nanotubes in 1991. Carbon nanotubes (CNT) have attracted and the ability to improve the structure and exceptional physical properties. CNTs have a high aspect ratio and low mass density that make ideal reinforcement filler for nanocomposite materials [22-24]. Multi-walled carbon nanotubes (MWCNTs) have high electrical conductivity, compared with other conductive nanofillers such as metal flakes [25]. MWCNTs thermoplastics composites have the ability to improve the stiffness, the thermal and mechanical Properties of 
unfilled polymeric composites [26]. In previous studies, some researchers were investigated to improve mechanical properties of polyethylene terephthalate (PET) by using low contents MWCNTs [27]. This work aims to improve the chemical, mechanical and thermal properties of PET by using a high content of MWCNTs filler.

\section{Materials and Methods}

\subsection{Materials}

In this research, we are applied poly (ethylene terephthalate) as granular form, and the density is $1.68 \mathrm{gm}$ per $\mathrm{ml}$ at $25^{\circ} \mathrm{C}$ was supplied from Sigma-Aldrich Company. UK. Multiwalled Carbon Nanotubes(MWCNTs) materials, Purity $99 \%$, length $(5 \mu \mathrm{m})$ and diameter $(15 \mathrm{~nm})$ were provided from Ad-Nano Technologies Private Limited, India.

\subsection{Preparation of Carboxylic Groups Functionalized MWCNTS}

$5 \mathrm{~g}$ of MWCNTs were refluxed in $100 \mathrm{ml}$ nitric acid $(70 \%)$ and $25 \mathrm{ml}$ sulfuric acid on bottom flask for overnight to create carboxyl functional groups of MWCNTs as shown in figure 1. After that, the Functionalized Multi-Walled Carbon Nanotubes were filtered and washed with deionized distilled water to get $\mathrm{PH}=7$. After the filtration, MWCNTs were dried at $70^{\circ} \mathrm{C}$ a vacuum oven overnight.

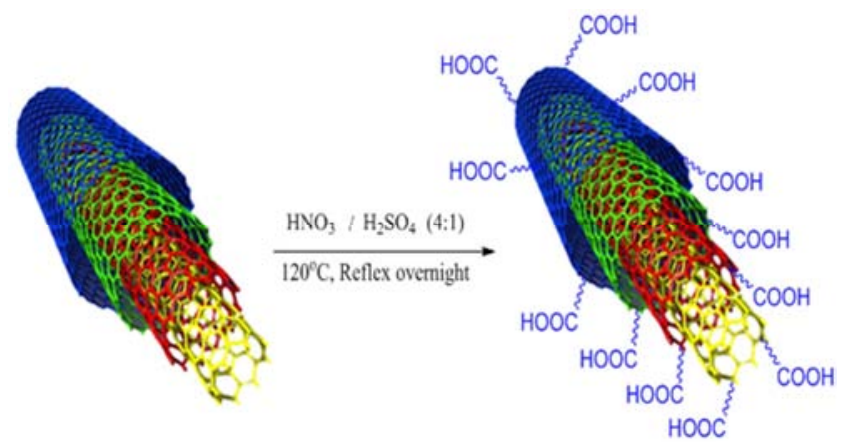

Fig. 1. Chemical modification of MWCNTs.

\subsection{Synthesis of PET-MWCNTs Composites}

Different weights $(0.1,0.5$, and $1 \%)$ wt $\%$ from functionalized MWCNTs were prepared by melting mixing technique with PET. The mixing method was applied in two stages. The first stage, the MWCNT/PETs powder was placed inside the chamber and mixed for 10 min at $25 \mathrm{rpm}$. The mixing speed was then increased to 70 rpm for 5 min more. The PET/MWCNTs composite material was then laminated by compression Aluminum molding into thin sheets of $80 \times 80 \times 5 \mathrm{~mm}$ and then left in a vacuum oven in $170^{\circ} \mathrm{C}$ annealing to allow of removing any bubbles from samples.

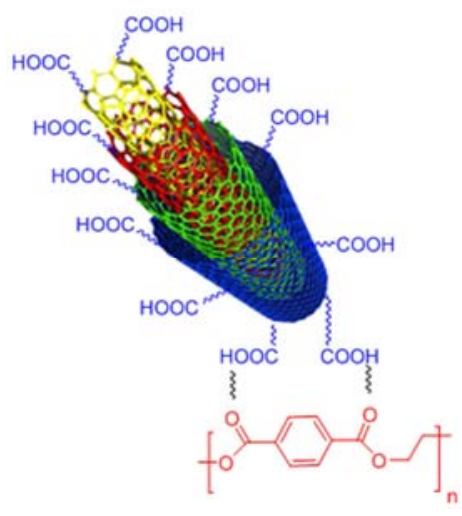

Fig. 2. Schematic showing possible interactions between the carboxylic group functionalized -MWCNTs fillers and the PET matrix.

\subsection{Fourier-Transform Infrared (FTIR) Tests}

The PET nanocomposites samples were tested by Perkin Elmer FTIR spectrophotometer within the wavelength range (4000-700 $\left.\mathrm{cm}^{-1}\right)$ to calculate the intensity of absorbance for carbonyl and hydroxyl indexes in the bands $1723 \mathrm{~cm}^{-1}$ and $3400 \mathrm{~cm}^{-1}$ of the samples according to the equations (1) and (2) [28].

$$
\begin{aligned}
& \text { Crabonyl index }=\frac{I \text { abs in } 1623 \mathrm{~cm}^{-1}}{I \text { abs in } 1958 \mathrm{~cm}^{-1}} \\
& \text { Hydroxyl index }=\frac{I \text { abs in } 3400 \mathrm{~cm}^{-1}}{I \text { abs in } 1958 \mathrm{~cm}^{-1}}
\end{aligned}
$$

\subsection{Thermogravimetric Analysis (TGA)}

Thermogravimetric Instrument (Perkin-Elmer's) company was used to follow the thermal stability of pure PET and PET nanocomposites. The heating rate of samples was about $10^{\circ} \mathrm{C}$ $\min ^{-1}$ in the range $20-600^{\circ} \mathrm{C}$. The loss of mass was followed in response to increasing temperature.

\subsection{Mechanical Tests}

Tensile strength tests were carried out by using A Tensile Universal Tester type AGS-X according to ASTM D 638. The samples were placed vertically in the jaws, and a tensile load was applied to pull out. The testing speed of samples was $10 \mathrm{~mm} / \mathrm{min}$.

\subsection{Scanning Electron Microscopy (SEM)}

The surface morphology of PET nanocomposites samples was examined under Scanning Electron Microscope (SEM) instrument model (HITACHI S-3000N). The samples were coated with $4 \mathrm{~mm}$ a gold layer and then the results were investigated.

\section{Results and Discussion}

\subsection{Fourier Transformed Infrared Spectroscopy (FTIR) Analysis}

FTIR tests were applied to confirm the presence of hydroxyl and carboxyl groups and to calculate the hydroxyl and 
carbonyl indexes of PET nanocomposites [29]. The obtained data from FT-IR spectra of the PET nanocomposites are shown in figure 3 within the range $700-4000 \mathrm{~cm}^{-1}$. Fig. 3 explains comparison the absorbance of pure PETand PET-MWCNTs composites with different ratios versus wavelengths. The spectra of FTTR proved the presence of the absorbance band at 3400 due to hydroxyl groups and the band $\left(1723 \mathrm{~cm}^{-1}\right)$ due to carbonyl groups that formed from carboxyl functional groups. From fig.4, it focused on the absorbance of hydroxyl and carbonyl bands within the range $\left(2000-1000 \mathrm{~cm}^{-1}\right)$ wavelengths. The increasing of incorporation of MWCNTs in pure PET between (0.1- 1\%) were attributed to decrease the intensity of absorbance of the hydroxyl and carbonyl indexes in the bands $3400 \mathrm{~cm}^{-1}$ and $1723 \mathrm{~cm}^{-1}$ as shown in fig.5. The characteristic of peaks observed at 1085,1192 , and $1723 \mathrm{~cm}^{-1}$, respectively, for the $\mathrm{COOH}-$ MWCNTs, were attributed to the stretching of the carboxylic groups and enhanced the interaction between pure PET chains. MWCNTs-PET composites. These results indicate that carboxylic groups were more interaction on the pure PET surface.

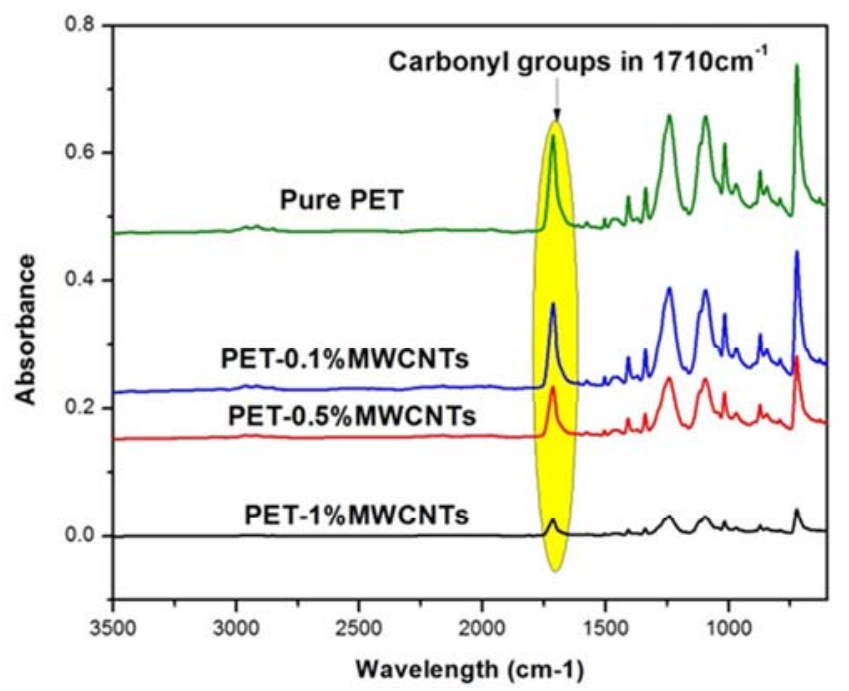

Fig. 3. FTIR absorbance of PET and different PET-MWCNTs samples.

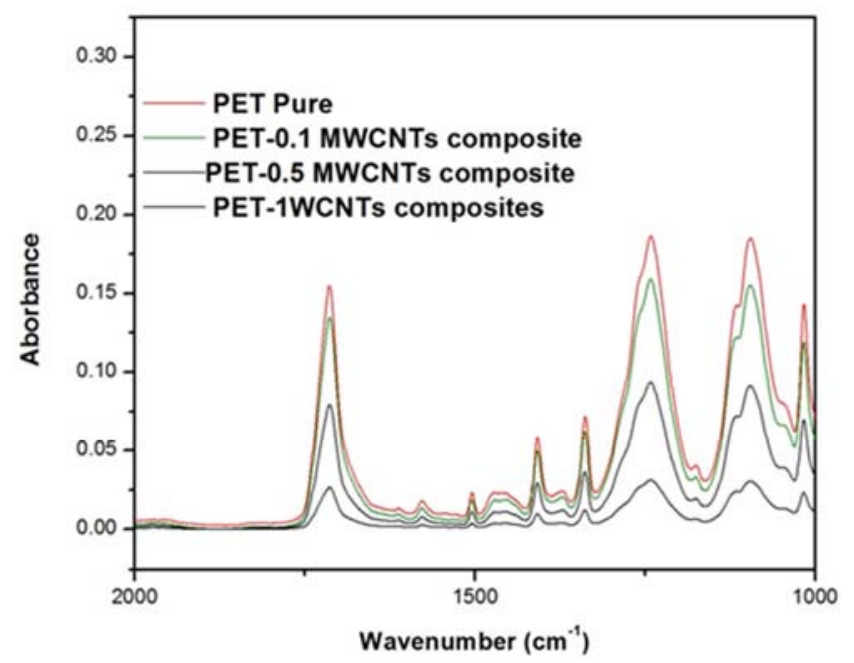

Fig. 4. FTIR absorbance of PET and PET-MWCNTs samples of the range 2000-1000 $\mathrm{cm}^{-1}$ wavelengths.

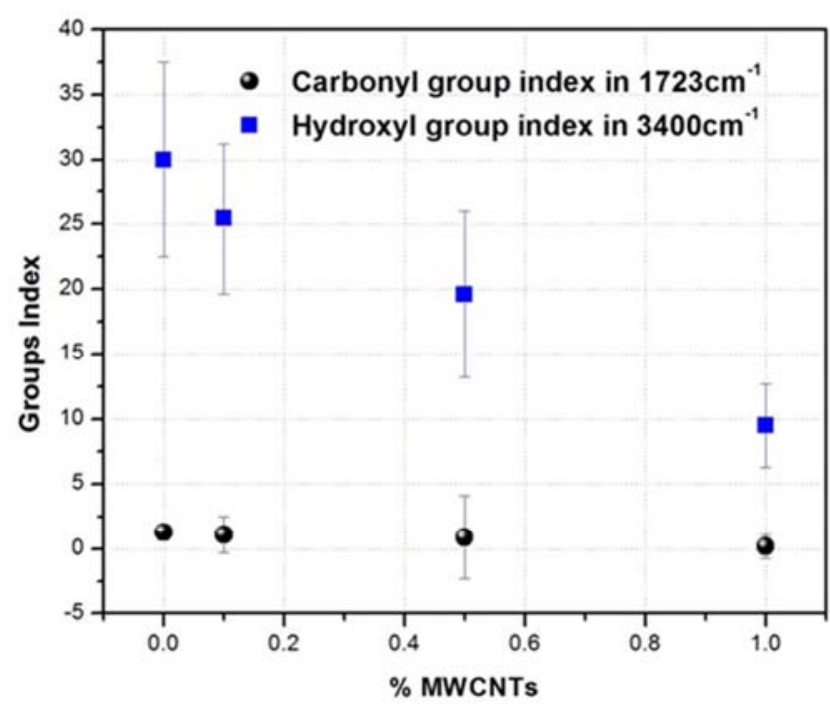

Fig. 5. Hydroxyl and carbonyl indexes of PET and PET-MWCNTs samples of the range $2000-1000 \mathrm{~cm}^{-1}$ wavelengths.

\subsection{Thermal Degradation Analysis of PET Nanocomposites}

Thermal degradation behavior of the PET pure and PETMWCNTs samples are shown in Figure 6, and $T_{\text {onset }}$ refers to the initial degradation temperature and is a relevant factor indicating on the thermal stability [30]. The maximum degradation temperature $\left(\mathrm{T}_{\max }\right), \mathrm{T}_{\text {onset }}$ and residual yield $\%$ at $600^{\circ} \mathrm{C}$ are summarized in Table 1 . The patterns of the curves for the pure PET sample are similar to that of PET-MWCNTs composites samples. From fig.1, it was observed that the thermal decomposition of samples was decreased with the increasing of the MWCNT contents from $(0.1-1 \%)$. The presence of the MWCNTs fillers is to make more stability of the PET matrix and gives good interfacial adhesion and higher crosslinking between the MWCNTs fillers and the PET pure. Table 1 shows that $\mathrm{T}_{\text {onset, }} \mathrm{T}_{\max }$, and residual yield is increased with the increasing the concentration of MWCNTs. The increasing in MWCNTs fillers in pure PET exhibited higher thermal stability by comparing with pure PET. $\mathrm{T}_{\max }$ of pure PET was $440^{\circ} \mathrm{C}$ and residual yield about $19.5 \%$ while PET$1 \%$ MWCNTs composites were $\mathrm{T}_{\max }$ about $488^{\circ} \mathrm{C}$ and residual yield is $35 \%$ as shown in table1. These values in Table 1 proved that MWCNTs fillers were accelerated by the high protecting effect against the thermal decomposition and enhanced the thermal stability of PET. These indicate that the size of crosslinking of MWCNTs are more than the of bond break of pure PET. In the final stage, the mass loss of PET-MWCNTs is less than the weight loss of pure PET.

Table 1. TGA thermograms degradation results of PET pure and PETMWCNTs composites.

\begin{tabular}{llll}
\hline System\% & $\mathbf{T}_{\text {onset }^{ \pm 0.1}}\left({ }^{\mathbf{0}} \mathbf{C}\right)$ & $\mathbf{T}_{\mathbf{m a x}} \mathbf{\pm 0 . 1}\left({ }^{\mathbf{0}} \mathbf{C}\right)$ & Residual yield (\%) \\
\hline PET control & 396 & 440 & 19.5 \\
0.1 MWCNTs & 401 & 458.5 & 22 \\
0.5MWCNTs & 405.7 & 465.7 & 24.8 \\
1MWCNTs & 418 & 488 & 35 \\
\hline
\end{tabular}




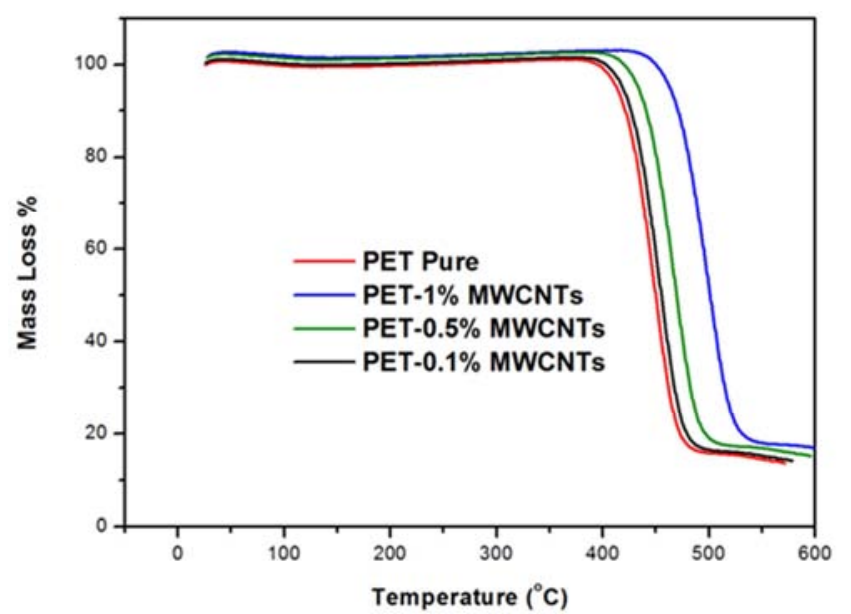

Fig. 6. TGA thermograms of PET nanocomposites.

\subsection{Mechanical Tests Analysis of PET Nanocomposites}

The tests of mechanical properties can reflect and evaluate the effects of adding MWCNTs fillers in pure PET [31]. It is evident from figure 7 and table 2 that tensile strength increases with adding a high amount of MWCNTs fillers to pure PET. The table 2 shows that the high content $(1 \%$ MWCNTs) increased to $56.3 \mathrm{MPa}$ comparing with pure PET $40.5 \mathrm{MPa}$. As in the case of the modulus of elasticity as shown in Table 1 that appeared a significant increase 1200 $\mathrm{MPa}$ with adding $1 \%$ MWCNTs fillers, whereas pure PET $500 \mathrm{MPa}$. The results of elongation at break in Table 2 indicate that a higher value of MWCNTs (1\%) inhibits the increase in elongation at break to $8.6 \%$, compared to pure PET $15.5 \%$.

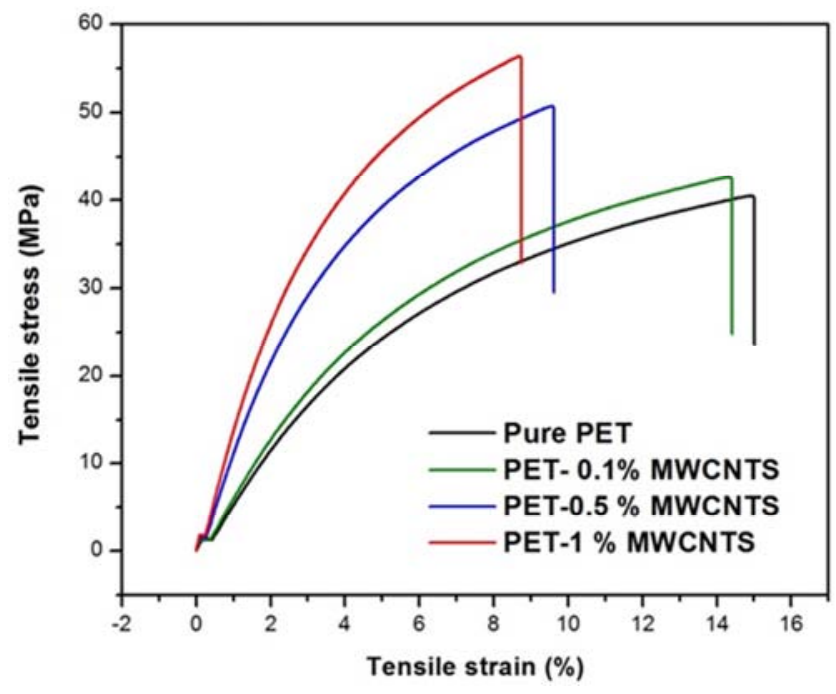

Fig. 7. Tensile Stress - Strain Curves of PET nanocomposites.

Table 2. The results from Tensile tests of PET nanocomposites.

\begin{tabular}{llll}
\hline System\% & Tensile Strength MPa(SD) & Modulus of Elasticity MPa(SD) & Strain at break\% (SD) \\
\hline PET control & $40.5(5.2)$ & $500(22)$ & $15.5(0.5)$ \\
0.1 MWCNTs & $42.6(0.9)$ & $540(18)$ & $14.2(0.3)$ \\
0.5MWCNTs & $50.4(1.4)$ & $1130(32)$ & $9.5(0.1)$ \\
1MWCNTs & $56.3(4.2)$ & $1200(35)$ & $8.6(0.6)$ \\
\hline
\end{tabular}

SD (Standard Deviation)

\subsection{Structural Changes on the Surface of PET Nanocomposites (SEM)}

The SEM micrographs of Pure PET and PET-MWCNTs composites $1 \%$ are shown in figure 8 and 9 respectively. MWCNTs composites micrograph has more dispersed and homogeneous between the molecules because of the great interface and high crosslinked between the pure PET chains while pure PET SEM figure observed more bristles and the interface between the chains with more space. The incorporation of $1 \% \mathrm{MWCNTs}$ in pure PET appeared higher crystallinity over the cross-section of PET.

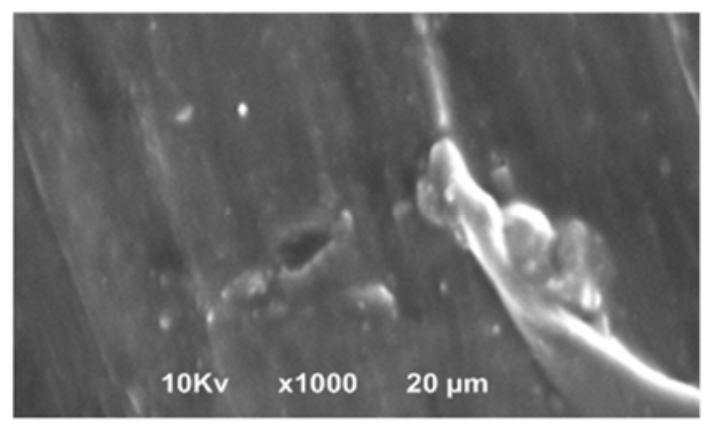

Fig. 8. SEM micrographs of pure PET.

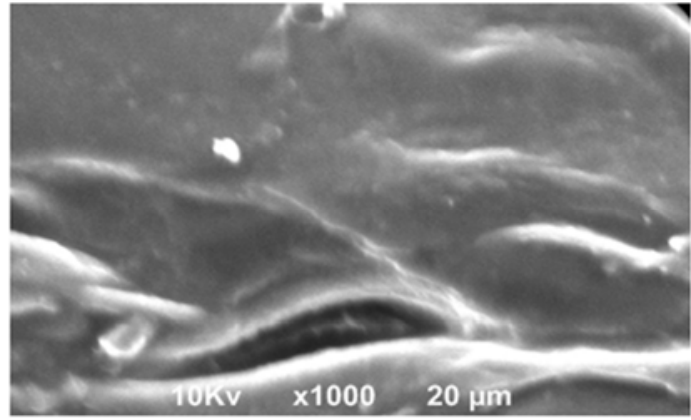

Fig. 9. SEM micrographs of PET-1\%MWCNTs composites.

\section{Conclusion}

In conclusion, increasing the concentrations of MWCNTs fillers from $0.1-1 \%$ enhanced in decreasing the intensities of absorbance in hydroxyl and carbonyl indexes in the bands $3400 \mathrm{~cm}^{-1}$ and $1715 \mathrm{~cm}^{-1}$, comparing with the intensity of pure PET that was increased in the same indexes. The higher content of MWCNTs fillers in pure PET made more thermal stability as result of the high crosslink density with of pure PET because the MWCNTs increased the crosslink density between the pure PET. It was clear that $1 \%$ wt MWCNTs 
improved the mechanical properties compared to other MWCNTs contents and pure PET.

\section{Acknowledgments}

The University of Al-Anbar-Iraq and Al-Anbar Technical Institute have supported this work, and I'd like to acknowledge the University of Technology of supporting this research about the chemical instruments.

\section{References}

[1] Iijima S. Nature, 1991, 354:56-8.

[2] Moniruzzaman M, Winey KI. Macromolecules, 2006, 39:5194-205.

[3] Marcio R. L, Luiz A. F. C, Sergio H. P, Sandro C. A, Materials Research, 2008, 11:347-352.

[4] Tsai T. Y, Li C. H, Chang C.H, Cheng W.H, Hwang C.L, Wu R.J. Adv. Mater, 2005, 17:1769-1773.

[5] Chang J. H, Kim S. J, Joo Y. L, Im S. Polymer, 2004, 45:919-926.

[6] Pegoretti A, Kolarik J, Peroni C, Migliaresi C. Polymer, 2004, 45:2751-2759.

[7] Imai $\mathrm{Y}$, Nishimura $\mathrm{S}$, Abe E, Tateyama H, Abiko A, Yamaguchi A, Taguchi H. J.Chem. Mater, 2002, 14:477-479.

[8] Supawee M, Wanlop H, Energy Procedia, 2014:547-553.

[9] Villmow T, Pegel S, Potschke P, Wagenknecht U, Compos. Sci. Technol, 2008, 68:777-789.

[10] Logakis E, Pollatos E, Pandis C, Peoglos V. I, Zuburtikudis C, Delides G, Vatalis A, Gjoka M, Syskakis E, Viras K, Compos. Sci. Technol, 2010, 70:328-335.

[11] Saeedeh M, Abdellah A, Charles D, 2010 Nano Mater, 2010, 78-83.

[12] Jeol S, Fenouillot F, Rousseau A, Varlot K. M. Macromolecules, 2007, 40:3229-3237.
[13] Giannelis E. P, Adv. Mater, 1996, 8:29-35.

[14] Rui Y, Xu M, Chin Z. J. Polym. Sci, 2014, 32:230-235.

[15] May-Pat A, Aviles F, Toro P. M, Yazdani-Pedra M, CauichRodríguez J. V. Express. Polym. Lett, 2012, 6:96-106.

[16] Kazushi Y, Supaphorn T. Advances in Materials Physics and Chemistry, 2013, 3:327-331.

[17] Sabine G, René F, Myrtill S. 2012, The Nano Trust Dossiers.

[18] Iman T, Abdelhossein F, Ali M. J. Steel and Composites Structure, 2014, 6: 825-834.

[19] Bien D. Ch, Bao Q. N, Le-Thu T. N, Ha T. N, Viet Q. N, Thang V. L, Nieu H. N. Chem. Cent. J, 2015, 9:10.

[20] Eduard A, Codrin D, Cristina S. Materials,2009, 2:2095-2153.

[21] Marcio R. L, Luiz A. F. C, Compos. J, 2008, 3 347-352.

[22] Nathalie G. V, Antxon M, Sebastián M, Pere C, María T. Compos. Sci. Technol, 2010, 70:789-796.

[23] Chao X. L, Jin W. Ch, Nanomaterials, 2012, 2: 329-349.

[24] Ager R. J, Kleina P. J, Lagoudasa D. C, Zhangb Q. L, Daib L, Baurc J. W. Compos. Sci. Technol, 2009, 69:898-904.

[25] Eduard A. S, Codrin D, Cristina S. Materials, 2009, 2:2095-2153.

[26] Barbes L, Radulescu C, Stihi C. Romanian Reports in Physics, 2014, 66:765-777.

[27] Moniruzzaman M, Winey K. I. Polymer Nanocomposites Containing Carbon Nanotubes. Macromolecules, 2006, 39:5194-205.

[28] Maryudi M, Anwaruddin H, Rosil M. Y, Beg D. H. International Journal of Engineering Research and Applications, 2013, 3:1156-1165.

[29] Bimestre B. H, Saron C. Materials Research, 2012, 15:467472.

[30] Bimestre B. H, Saron. C. Materials Research, 2012, 15:467-72.

[31] Zaman H. U, Hun P.D, Khan R. A, Yoon K. B. Fullerenes, Nanotubes and Carbon Nanostructures, 2013, 21:701-11. 